\title{
THE SYMMETRY ALGEBRA AND CONSERVED CURRENTS FOR KLEIN-GORDON EQUATION ON QUANTUM MINKOWSKI SPACE
}

\author{
MAŁGORZATA KLIMEK \\ Institute of Mathematics and Computer Science \\ Technical University of Czestochowa \\ ul. Dabrowskiego 73, 42-200 Częstochowa, Poland \\ E-mail: klimek@matinf.pcz.czest.pl
}

\begin{abstract}
The symmetry operators for Klein-Gordon equation on quantum Minkowski space are derived and their algebra is studied. The explicit form of the Leibniz rules for derivatives and variables for the case $Z=0$ is given. It is applied then with symmetry operators to the construction of the conservation law and the explicit form of conserved currents for Klein-Gordon equation.
\end{abstract}

1. Introduction. The existence of currents fulfilling the conservation laws is the important feature of invariant models in classical mechanics and field-theory. They can be derived by Noether theorem where each symmetry of the action yields the connected conserved current. In the case of linear equations of motion with constant coefficients (such as for example Klein-Gordon and Dirac equations) the procedure was simplified by Takahashi and Umezawa [10]. We have studied the extension of this procedure to the discrete models with generalized difference derivatives [1-3]. It appeared that after modifying the Leibniz rule for this calculus we were able to construct the conservation laws and constants of motion for this type of models. In the presented paper we would like to show that the extension also works for Klein-Gordon equation on quantum Minkowski space

$$
\left(\square+m^{2}\right) \Phi=0
$$

where the d'Alembert operator looks as follows

$$
\square=g_{i j} \partial^{i} \partial^{j}
$$

1991 Mathematics Subject Classification: Primary 53Z05; Secondary 35Q99, 57T05, 81Q99. Research supported by KBN grant 2P 30208706 .

The paper is in final form and no version of it will be published elsewhere. 
This equation was constructed by Podleś [7] within the framework of covariant differential calculi and exterior algebras on quantum homogenous spaces endowed with the action of quantum Poincaré group $[8,9]$.

The fundamental rules for derivatives and variables are given by the formulae:

$$
\begin{gathered}
\partial^{j}\left(x^{i}\right)=g^{j i} \\
\partial^{l} \partial^{k}=R^{l k}{ }_{i j} \partial^{i} \partial^{j} \\
\partial^{i} x^{k}=g^{i k}+\left(R^{i k}{ }_{a b} x^{a}-(R Z)_{b}^{i k}\right) \partial^{b} \\
(R-1)^{i j}{ }_{k l}\left[x^{k} x^{l}-Z_{s}^{k l} x^{s}+T^{k l}\right]=0
\end{gathered}
$$

with R-matrix fulfilling QYBE $R_{23} R_{12} R_{23}=R_{12} R_{23} R_{12}$ and the condition $R^{2}=1$.

As we have mentioned in the construction of conservation law and explicit form of currents we shall follow the classical procedure proposed by Takahashi and Umezawa for linear differential equations in field theory. However, in the classical case the Leibniz rule for commutative differential calculus is known. Thus our investigation shall include the description of Leibniz rules for noncommutative differential calculus (3). The construction consists of the following steps:

- we need solutions of Klein-Gordon equation. They were obtained by Podleś [7] as formal power series of noncommuting variables. He considered two cases $R=\tau$ and $Z=0$

- then we describe and modify Leibniz rule for derivatives in the noncommutative differential calculus on quantum Minkowski space (section 2 and 4)

- we construct the symmetry operators of Klein-Gordon equation and study their algebra (section 3)

- the symmetry operators and modified Leibniz rule for derivatives are applied in the conservation law and explicit formulae for conserved currents (section 4).

In the paper we shall study the case $Z=0$, the general case will be presented in the next paper [4].

2. The Leibniz rules in differential calculi on quantum Minkowski spaces. The condition $Z=0$ yields the following rules for the variables and derivatives:

$$
\begin{gathered}
\partial^{i} \partial^{j}=R_{k l}^{i j} \partial^{k} \partial^{l} \\
\partial^{j} x^{k}=g^{j k}+R^{j k}{ }_{a b} x^{a} \partial^{b} \\
x^{i} x^{j}=R_{k l}^{i j}{ }_{k} x^{k}+v^{i j}
\end{gathered}
$$

where the metric tensor $g$ is $R$-symmetric and $v=R T-T$ is $R$-antisymmetric:

$$
R g=g \quad R v=-v
$$

In order to study the explicit form of Leibniz rules for products of functions understood as formal power series of monomials we denote an arbitrary monomial as follows:

$$
\left[k_{1}, k_{2}, \ldots, k_{n}\right]=x^{k_{1}} x^{k_{2}} \ldots x^{k_{n}}
$$

where the indices $k_{i}$ denote the number of variable. 
Let us notice that the property of the covariant differential calculus (1.10) from [7] yields the Leibniz rule for derivatives:

$$
\partial^{i} f g=\left(\partial^{i} f\right) g+(\zeta f)_{j}^{i} \partial^{j} g
$$

where the transformation operator obeys the rule:

$$
[\zeta(f g)]_{j}^{i}=(\zeta f)_{m}^{i}(\zeta g)_{j}^{m}
$$

The result of taking the derivative of function written down for monomials looks as follows

$$
\left(\partial^{i}\left[k_{1}, \ldots, k_{n}\right]\right)=\sum_{l=1}^{n}\left(\zeta\left[k_{1}, \ldots, k_{l-1}\right]\right)_{j}^{i} g^{j k_{l}}\left[k_{l+1}, \ldots, k_{n}\right]
$$

with the initial and the last term of the form:

$$
l=1 \quad g^{i k_{1}}\left[k_{2}, \ldots, k_{n}\right] \quad l=n \quad\left(\zeta\left[k_{1}, \ldots, k_{n-1}\right]\right)_{j}^{i} g^{j k_{n}}
$$

The expression (9) is reformulation of the formula given in [7] written down using the introduced transformation operator $\zeta$ which for the case $Z=0$ acts on monomials as follows:

$$
\left(\zeta\left[k_{1}, k_{2}, \ldots, k_{n}\right]\right)_{j}^{i}=R_{m_{1} \alpha_{1}}^{i k_{1}} R_{m_{2} \alpha_{2}}^{\alpha_{1} k_{2}} R_{m_{n j} j}^{\alpha_{n-1} k_{n}}\left[m_{1}, m_{2}, \ldots, m_{n}\right]
$$

This formula determines also the way it transforms an arbitrary function on quantum Minkowski space expanded into formal power series:

$$
f(x)=\sum a_{k_{1} k_{2} \ldots k_{n}}\left[k_{1}, k_{2}, \ldots, k_{n}\right] \quad(\zeta f)_{j}^{i}=\sum a_{k_{1} k_{2} \ldots k_{n}}\left(\zeta\left[k_{1}, k_{2}, \ldots, k_{n}\right]\right)_{j}^{i}
$$

The analogous Leibniz rule holds also for the variable $x^{i}$ :

$$
x^{i} f g=\left(x^{i} f\right) g=\left(x^{i} f\right)_{a c t} g+(\zeta f)_{j}^{i} x^{j} g
$$

where the action of variable on monomial is determined by properties of quantum Minkowski space (6):

$$
\left(x^{i}\left[k_{1}, \ldots, k_{n}\right]\right)_{a c t}=\sum_{l=1}^{n}\left(\zeta\left[k_{1}, \ldots, k_{l-1}\right]\right)_{j}^{i} v^{j k_{l}}\left[k_{l+1}, \ldots, k_{n}\right]
$$

with the following initial and last term

$$
l=1 \quad v^{i k_{1}}\left[k_{2}, \ldots, k_{n}\right] \quad l=n \quad\left(\zeta\left[k_{1}, \ldots, k_{n-1}\right]\right)_{j}^{i} v^{j k_{n}}
$$

In the case when the quantum variables $R$-commute $x^{i} x^{j}=R_{k l}^{i j} x^{k} x^{l}$ the Leibniz rule for variables reduces to the formula:

$$
x^{i} f g=\left(x^{i} f\right) g=(\zeta f)_{j}^{i} x^{j} g
$$

in which no selfinteraction of variables term appears.

The Leibniz rules $(7,13)$ can be written in the vector form:

$$
\begin{gathered}
\vec{\partial} f g=(\vec{\partial} f) g+(\zeta f) \vec{\partial} g \\
\vec{x} f g=(\vec{x} f) g=(\vec{x} f)_{a c t} g+(\zeta f) \vec{x} g
\end{gathered}
$$

where the $\zeta$ operator acting on the scalar function gives matrix.

The Leibniz rules for derivatives and variables require the introduction of the new operator $\zeta$ which does not appear in classical differential calculus. Let us notice however that this kind of deformation of Leibniz rules is also characteristic for discrete calculus 
of generalized difference derivative on functions of commuting variables [1-3]. The form of the transformation operator is then different from (11) but nevertheless the rule looks very similar to (7). In section 4 we use this analogy in modification of the Leibniz rule and its application to the conservation law.

3. The symmetry operators for Klein-Gordon equation and their algebra. In this section we shall look for operators commuting with the operator of Klein-Gordon equation. These operators transform a solution into solution.

In the calculations we have used the followig splitting of the Kronecker delta:

$$
\begin{gathered}
\frac{1}{2}()^{a b}{ }_{c d}+\frac{1}{2}[]^{a b}{ }_{c d}=\delta^{a b}{ }_{c d} \\
()^{a b}{ }_{c d}=\left(\delta^{a b}{ }_{c d}-R^{a b}{ }_{c d}\right) \quad[]^{a b}{ }_{c d}=\left(\delta^{a b}{ }_{c d}+R^{a b}{ }_{c d}\right)
\end{gathered}
$$

As we see the symbol ( $)^{a b}{ }_{c d}$ denotes the $R$-antisymmetric part of splitting and correspondingly [ $]_{c d}^{a b}$ the $R$-symmetric one.

The set of symmetry operators can be divided analogously to the classical case into two parts - momenta $P^{i}$ ( as shown in [7]) and the angular momentum $M^{i j}$. These operators are defined by the following formulae:

$$
\begin{gathered}
P^{i}=\partial^{i} \\
M^{i j}=()^{i j}{ }_{c d} x^{c} \partial^{d}=\left(\delta^{i j}{ }_{c d}-R^{i j}{ }_{c d}\right) x^{c} \partial^{d}
\end{gathered}
$$

Both these operators commute with the d'Alembert operator (2) and therefore also with the Klein-Gordon equation. The angular momentum (22) is $R$ - antisymmetric and traceless operator:

$$
R_{k l}^{a b} M^{k l}=-M^{a b} \quad g_{a b} M^{a b}=0
$$

In the sequel we apply the following formulae for $R$ - symmetric and $R$ - antisymmetric symbols resulting from QYBE and the assumption $R^{2}=1$ :

$$
\begin{gathered}
()^{a b}{ }_{c d} R^{y c}{ }_{s t} R^{t d}{ }_{m n}[]^{s m}{ }_{k l}=0 \\
()^{i j}{ }_{y z} R^{z a}{ }_{k l} R^{l b}{ }_{s t} R^{y k}{ }_{p \gamma} R^{\gamma s}{ }_{T V}[]^{V t}{ }_{m n}=0 \\
()^{a b}{ }_{c d} R^{y c}{ }_{s t} R^{t d}{ }_{m n} M^{s m}=2 R^{y a}{ }_{A t} R^{t b}{ }_{B n} M^{A B} \\
()^{i j}{ }_{y z} R^{z a}{ }_{k l} R^{l b}{ }_{s t} R^{y k}{ }_{p \gamma} R^{\gamma s}{ }_{T V} M^{p T} M^{V t}=2 R^{j a}{ }_{k l} R^{l b}{ }_{s t} R^{i k}{ }_{p \gamma} R^{\gamma s}{ }_{T V} M^{p T} M^{V t}
\end{gathered}
$$

Using the above formulae we derive the commutation rules :

- for momenta $P^{i}=\partial^{i}$

$$
P^{a} P^{b}-R^{a b}{ }_{c d} P^{c} P^{d}=0
$$

- for momentum and angular momentum operator

$$
P^{y} M^{a b}-R_{k l}^{y a} R^{l b}{ }_{s t} M^{k s} P^{t}=()^{a b}{ }_{c d} g^{y c} P^{d}
$$

- for components of the angular momentum

$$
M^{i j} M^{a b}-R^{j a}{ }_{k l} R^{l b}{ }_{s t} R^{i k}{ }_{p \gamma} R^{\gamma s}{ }_{T V} \quad M^{p T} M^{V t}=\Gamma_{T t}^{i j, a b} P^{T} P^{t}+\Lambda_{T t}^{i j, a b} M^{T t}
$$

where the constant coefficients look as follows

$$
\Gamma_{T t}^{i j, a b}=()^{i j}{ }_{y z} R^{z a}{ }_{k l} R^{l b}{ }_{s t}()^{k s}{ }_{p T} v^{y p}
$$




$$
\Lambda_{V t}^{i j, a b}=()^{i j}{ }_{V z}()^{a b}{ }_{c t} g^{z c}
$$

In terms of $R$-commutators the algebra of symmetry operators is similar to the classical Poincaré algebra with exception of the term quadratic in momenta which is due to the selfinteraction of variables (6).

We can as well rewrite the algebra with $R$-commutators $(25,26,27)$ using the Lie brackets and then we obtain the algebra quadratic in all operators.

For the set of symmetry operators we can consider also the Leibniz rules. It was described in section 2 for momenta (7). Additionaly using the Leibniz rule derived for variables (13) we obtain the Leibniz rule for the angular momentum operator (we denote the product as $f g=f \otimes g)$

$$
M^{i j}(f \otimes g)=M^{i j} f \otimes g+\zeta_{\beta}^{i} \zeta_{\alpha}^{j} f \otimes M^{\beta \alpha} g+()^{i j}{ }_{a b} \tilde{\zeta}^{a} \zeta_{\alpha}^{b} f \otimes \partial^{\alpha} g
$$

with the additional operator $\tilde{\zeta}$ (being the result of selfinteraction of variables) acting as follows on monomials:

$$
\begin{gathered}
\tilde{\zeta}^{a}\left[k_{1}, \ldots, k_{n}\right]=\left(x^{a}\left[k_{1}, \ldots, k_{n}\right]\right)_{a c t}=\sum_{l=1}^{n}\left(\zeta\left[k_{1}, \ldots, k_{l-1}\right]\right)_{j}^{a} v^{j k_{l}}\left[k_{l+1}, \ldots, k_{n}\right] \\
l=1 \quad v^{a k_{1}}\left[k_{2}, \ldots, k_{n}\right] \quad l=n \quad\left(\zeta\left[k_{1}, \ldots, k_{n-1}\right]\right)_{j}^{a} v^{j k_{n}}
\end{gathered}
$$

We have investigated the algebraic properties of symmetry operators and derived for them Leibniz rules. The problem whether they define also the coalgebraic structure is open and needs further study.

4. The conservation law for Klein-Gordon equation. Now we apply the Leibniz rule for derivatives (7) in construction of the conservation law. In this deformed formula we have obtained on the right-hand side operator acting on both functions of the product. At this point it is usefull to remind the Leibniz rule of discrete calculus of generalized difference derivative $[1,3]$ :

$$
\partial^{i} f g=\left(\partial^{i} f\right) g+(\zeta f)^{i} \partial^{i} g
$$

where the derivative is defined for functions of real variables as follows :

$$
\partial^{i} f(\vec{x})=\left[\phi^{i}\left(x^{i}\right)-x^{i}\right]^{-1}\left[\zeta^{i}-1\right] f(\vec{x})
$$

and the transformation operator acts as translation in the direction $i$ to the next point of the lattice $\zeta^{i} f(\vec{x})=f\left(x_{1}, \ldots, x_{i-1}, \phi_{i}\left(x_{i}\right), x_{i+1}, \ldots, x_{n}\right)$. In the discrete models described using the derivatives (30) we solved the problem modyfing the Leibniz rule to obtain on the right-hand side of (29) the operators acting only on one of the functions of the product $[1,3]$ :

$$
\partial^{i}\left(\zeta^{-i} f\right) g=\left(-\partial^{\dagger}{ }^{i} f\right) g+f \partial^{i} g
$$

The modification consists of changing the product on the left-hand side by the inverse operator which in the discrete calculus is of the form

$$
\zeta^{-i} f(\vec{x})=f\left(x_{1}, \ldots, x_{i-1}, \phi_{i}^{-1}\left(x_{i}\right), x_{i+1}, \ldots, x_{n}\right)
$$

what means that it translates the point to the previous one in the direction $i$ and of replacing one of the derivatives on the right-hand side with its conjugation $\partial^{\dagger} i=-\partial^{i} \zeta^{-i}$. 
Now the question arises whether in noncommutative differential calculus on quantum Minkowski space the inverse transformation operator exists. The answer for the studied case $Z=0$ is positive. Let us introduce the operator $\zeta^{-}$by its action on monomials:

$$
\left(\zeta^{-}\left[k_{1}, \ldots, k_{n-1}, k_{n}\right]\right)_{j}^{i}=R_{\alpha_{n-1} m_{n}}^{k_{n} i} R_{\alpha_{n-2} m_{n-1}}^{k_{n-1} \alpha_{n-1}} \underset{j m_{1}}{k_{1} \alpha_{1}}\left[m_{1}, \ldots, m_{n-1}, m_{n}\right]
$$

It is easy to check that its action on products of functions is analogous to the formula (8) describing it for the transformation operator

$$
\left(\zeta^{-} f g\right)_{j}^{i}=\left(\zeta^{-} f\right)_{j}^{m}\left(\zeta^{-} g\right)_{m}^{i}
$$

For the operators $\zeta$ and $\zeta^{-}$the following proposition is valid.

Proposition.

$$
\begin{gathered}
\zeta_{a}^{-j} \zeta_{j}^{i}=\zeta_{a}^{j} \zeta_{j}^{-i}=\delta_{a}^{i} \\
\zeta_{a}^{-j}=* \zeta_{a}^{j} *
\end{gathered}
$$

where *-operation acts on Minkowski space and derivatives as described in $[7,8]$.

P r o of. We shall show that formulae $(33,34)$ are valid on monomials and therefore by linearity of operators $\zeta, \zeta^{-}$and $* \zeta *$ they are fulfilled for arbitrary function on quantum Minkowski space. From associativity of quantum Minkowski space it follows that the monomials can be written as a products:

$$
\left[k_{1}, \ldots, k_{n-1}, k_{n}\right]=\left[k_{1}, \ldots, k_{n-1}\right]\left[k_{n}\right]
$$

Let us check the formula (33) for monomials of the first order:

$$
\zeta_{a}^{-j} \zeta_{j}^{i}[k]=R_{m j}^{i k} R_{a l}^{m j}[l]=\delta^{i k}{ }_{a l}[l]=[k] \delta_{a}^{i}
$$

We have used here the property $R^{2}=1$. Now we apply the mathematical induction principle assuming that the proposition holds for monomials of order $<n$. We check the formula for arbitrary monomials of order $n$ :

$$
\begin{gathered}
\zeta_{a}^{-j} \zeta_{j}^{i}\left[k_{1}, \ldots, k_{n-1}, k_{n}\right]=\zeta_{a}^{-j} \zeta_{j}^{i}\left[k_{1}, \ldots, k_{n-1}\right]\left[k_{n}\right]= \\
\left(\zeta_{a}^{-m} \zeta_{l}^{i}\left[k_{1}, \ldots, k_{n-1}\right]\right)\left(\zeta_{m}^{-j} \zeta_{j}^{l}\left[k_{n}\right]\right)=\left(\zeta_{a}^{-m} \zeta_{l}^{i}\left[k_{1}, \ldots, k_{n-1}\right]\right)\left[k_{n}\right] \delta_{m}^{l}= \\
\left(\zeta_{a}^{-m} \zeta_{m}^{i}\left[k_{1}, \ldots, k_{n-1}\right]\right)\left[k_{n}\right]=\left[k_{1}, \ldots, k_{n-1}\right]\left[k_{n}\right] \delta_{a}^{i}=\left[k_{1}, \ldots, k_{n-1}, k_{n}\right] \delta_{a}^{i}
\end{gathered}
$$

We have used the properties of operators $\zeta$ and $\zeta^{-}$with respect to the product of functions $(8,32)$. As the proposition holds for arbitrary monomial of order $n$ we conclude that by induction principle it is valid for all monomials and therefore for all functions expanded into formal power series. The proof of the second part of (33) is analogous to the presented.

In the proof of (34) we use the property of $R$ matrix [8]:

$$
\bar{R}_{a b}^{i j}=R_{b a}^{j i}
$$

Let us start with an arbitrary monomial of the first order and use $(31,36)$ :

$$
* \zeta_{a}^{j} *[k]=* \zeta_{a}^{j}[k]=* R_{m a}^{j k}[m]=[m]{\overline{R^{j k}}}_{m a}=R_{a m}^{k j}[m]=\zeta_{a}^{-}{ }^{j}[k]
$$

Now we assume that (34) holds for monomials of order $<n$ and use the property of transformation operator $(8,32)$. Then for monomial of order $n$ we get:

$$
* \zeta_{a}^{j} *\left[k_{1}, \ldots, k_{n}\right]=* \zeta_{a}^{j} *\left(\left[k_{1}, \ldots, k_{n-1}\right]\left[k_{n}\right]\right)=
$$




$$
\begin{gathered}
* \zeta_{a}^{j}\left[k_{n}\right] *\left[k_{1}, \ldots, k_{n-1}\right]=*\left(\zeta_{l}^{j}\left[k_{n}\right]\right) \zeta_{a}^{l} *\left[k_{1}, \ldots, k_{n-1}\right]= \\
\left(* \zeta_{a}^{l} *\left[k_{1}, \ldots, k_{n-1}\right]\right) *\left(\zeta_{l}^{j}\left[k_{n}\right]\right)=\left(\zeta_{a}^{-}{ }^{l}\left[k_{1}, \ldots, k_{n-1}\right]\right) \zeta_{l}^{-j}\left[k_{n}\right]=\zeta_{a}^{-j}\left[k_{1}, \ldots, k_{n}\right]
\end{gathered}
$$

We conclude that by induction principle (34) is valid for monomials of arbitrary order and therefore by linearity for arbitrary function on quantum Minkowski space expanded into formal power series.

We have proved the proposition for matrices fulfilling $R^{2}=1$ but (33) can be extended to invertible $R$-matrices. This is the case in the braided differential calculus $[5,6]$. Considering the Leibniz rule introduced there by Majid we conclude that on monomials it is similar to (7) and probably can be modified the way we propose in the formula (37). It seems therefore that the construction of conservation law and conserved currents would also be possible in models built within the framework of the braided differential calculi.

Now we can modify Leibniz rule (7) in order to obtain on the right-hand side operators acting only on one of the functions in the product :

$$
\partial^{i}\left(\zeta_{i}^{-a} f\right) g=\left(-\partial^{\dagger a} f\right) g+f \partial^{a} g
$$

where we use the notation $\partial^{\dagger a}=-\partial^{i} \zeta_{i}^{-a}=-\partial^{i} * \zeta_{i}^{a} *$.

The Leibniz rule for conjugated derivative looks as follows:

$$
\partial^{\dagger a} f g=\left(\partial^{\dagger i} f\right) \zeta_{i}^{-a} g+f \partial^{\dagger a} g
$$

Let us notice that we can identify the operator $\partial^{\dagger a}$ with $*\left(-\partial^{a}\right) *$. Indeed the Leibniz rule for this operator is the same as (38):

$$
\begin{gathered}
*\left(-\partial^{a}\right) * f g=*\left(-\partial^{a}\right)(* g)(* f)=*\left[-\left(\partial^{a} * g\right) * f-\left(\zeta_{i}^{a} * g\right) \partial^{i} * f\right]= \\
=\left(-* \partial^{i} * f\right) \zeta_{i}^{-}{ }^{a} g+f\left(-* \partial^{a} * g\right)
\end{gathered}
$$

We check the equality of these operators on arbitrary monomials of first order:

$$
*\left(-\partial^{a}\right) * x^{k}=-\overline{g^{a k}}=-g^{k a} \quad \partial^{\dagger a} x^{k}=-R^{k a}{ }_{i m} g^{i m}=-g^{k a}
$$

We have used the properties of the metric tensor: $R g=g$ and $\overline{g^{i j}}=g^{j i}$ from [7]. From Leibniz rules $(38,39)$ and from the equality of action of both operators on monomials of the first order (40) we see that application of the induction principle yields the identical action of $\partial^{\dagger a}$ and $*\left(-\partial^{a}\right) *$ on monomials of arbitrary order. Thus they act in the same way on all the functions on quantum Minkowski space and therefore are identical:

$$
\partial^{\dagger a}=*\left(-\partial^{a}\right) *
$$

This identity we use in the sequel in the construction of conserved currents.

Let us consider the modified Leibniz rule (37) for the following expression:

$$
\partial^{i}\left[-\left(\zeta_{i}^{-a} \partial^{\dagger}{ }^{m} F\right) g_{m a} G-\left(\zeta_{i}^{-a} F\right) g_{a m} \partial^{m} G\right]=\left(\square^{\dagger} F\right) G-F \square G
$$

where $F$ and $G$ are arbitrary functions on quantum Minkowski space.

Let us now choose the functions $F$ and $G$ as follows:

- the function $G$ is a transformed solution of Klein-Gordon equation

$$
G=\delta^{\omega} \Phi \quad\left(\square+m^{2}\right) \delta^{\omega} \Phi=0
$$


where the transformation $\delta^{\omega}$ is given by symmetry operators - the momentum or the angular momentum:

$$
\delta^{i}=\partial^{i} \quad \delta^{i j}=M^{i j}
$$

- the function $F=* \Phi$ where $\Phi$ is a solution of Klein-Gordon equation.

This function fulfills the conjugated Klein-Gordon equation:

$$
\begin{gathered}
\left(\square^{\dagger}+m^{2}\right) * \Phi=\left(\partial^{\dagger a} \partial^{\dagger m} g_{m a}+m^{2}\right) * \Phi= \\
=\left(* \partial^{a} * * \partial^{m} * g_{m a}+m^{2}\right) * \Phi=*\left(\partial^{a} g_{a m} \partial^{m}+m^{2}\right) \Phi=*\left(\square+m^{2}\right) \Phi=0
\end{gathered}
$$

The solutions $\delta^{\omega} \Phi$ and $* \Phi$ we insert into the currents. Let us notice that each symmetry operator allows to construct the conserved current:

$$
J_{i}^{\omega}=\left(\zeta_{i}^{-a} \partial^{\dagger m} * \Phi\right) g_{m a} \delta^{\omega} \Phi+\left(\zeta_{i}^{-a} * \Phi\right) g_{a m} \partial^{m} \delta^{\omega} \Phi
$$

which in consequence of (42) fulfills on-shell the following equation:

$$
\partial^{i} J_{i}^{\omega}=-\left(\square^{\dagger} * \Phi\right) \delta^{\omega} \Phi+(* \Phi) \square \delta^{\omega} \Phi=0
$$

The above formula yields on-shell the conservation law for Klein-Gordon equation on quantum Minkowski space:

$$
\partial^{i} J_{i}^{\omega}=0
$$

obeyed by currents defined in (46).

5. Final remarks. We have discussed the construction of the conservation law and conserved currents for Klein-Gordon equation connected with differential calculi fulfilling the condition $Z=0$.

The interesting problem is the extension of the presented method to an arbitrary linear equation of motion (including the Dirac equation on quantum Minkowski space [7]) and to the general differential calculus on quantum Minkowski space. After modification of the Leibniz rule for derivatives we can follow the analogy between the discrete and noncommutative models and construct the currents for equations on the quantum Minkowski space similarly to the general solution of the discrete problem given in [3]. The results will be presented in the next paper [4].

The other open problem is the understanding and the construction of constants of motion from conserved currents what requires the application of integral calculus on quantum Minkowski space and would be important for developing mechanical and fieldtheoretic models on noncommutative spaces.

Acknowledgments. The author is thankful to Professor J. Lukierski for fruitful discussions and to the referee for his valuable remarks.

\section{References}

[1] M. Klimek, J. Phys. A: Math. \& Gen. 26 (1993), 955.

[2] M. Klimek, in Papers of the $3^{\text {rd }}$ International Colloquium on Quantum Groups and Physics, Czechoslovak J.Phys. 44 (1994), 1049 
[3] M. Klimek, J. Phys. A: Math. \& Gen. 29 (1996), 1747.

[4] M. Klimek, in preparation.

[5] S. Majid, J.M.P. 34 (1993), 2045.

[6] S. Majid, J.M.P. 34 (1993), 4843.

[7] P. Podleś, Commun. Math. Phys. 181 (1996), 569.

[8] P. Podleś and S.L. Woronowicz, On the Structure of Inhomogenous Quantum Groups, hep-th 9412058, UC Berkeley preprint PAM 631, to appear in Commun. Math. Phys.

[9] P. Podleś and S.L. Woronowicz, Commun. Math. Phys. 178 (1996), 61.

[10] Y. Takahashi, An Introduction to Field Quantization, Pergamon Press, Oxford 1969 and references therein. 